\title{
ORIGINAL ARTICLE \\ Parental inconsistency, impulsive choice and neural value representations in healthy adolescents
}

\author{
S Schneider ${ }^{1}$, J Peters ${ }^{1,2}$, JM Peth $^{1}$ and C Büchel ${ }^{1,3}$
}

A well-characterized potential marker for addiction is impulsive choice, stably measured by delay discounting (DD) paradigms. While genetic influences partly account for inter-individual variance in impulsivity, environmental factors such as parenting practices may have an important role. The present study investigates how inconsistent fulfillment of delayed reward promises impacts on DD. A combined correlational and experimental functional magnetic resonance imaging (fMRI) design was performed in a sample of 48 healthy adolescents (13-15 years). More specifically, neural activation during a DD task was investigated at two assessment points $\left(T_{0}\right.$ and $\left.T_{1}\right)$. Adolescents' self-reports of parenting and substance use were assessed at $T_{0}$. Between assessment points, we experimentally varied the reliability of delayed reward promises, measuring the impact of this intervention on DD and neural value processing at $T_{1}$. In the correlational part, same-sex parent reward inconsistency was associated with steeper DD and an attenuated subjective value (SV) representation in the nucleus accumbens (NAcc) and ventromedial prefrontal cortex (vmPFC). Steeper DD was in turn associated with alcohol use during the past year. In the experimental part, the reward inconsistency manipulation resulted in an attenuation of the NAcc SV representation, similar to the parental inconsistency effect. Together, our correlational and experimental findings raise new light on how parents may influence their children's degree of impulsivity, making parenting a potential target in addiction prevention.

Translational Psychiatry (2014) 4, e382; doi:10.1038/tp.2014.20; published online 15 April 2014

\section{INTRODUCTION}

All parents sometimes promise future rewards to their children, and eventually, not all of these promises will be kept. However, some parents feel more obligated to let action follow words, while others are more forgetful or careless. If promised delayed rewards are regularly omitted, children may struggle to reliably estimate the value of delayed rewards and become more inclined to turn toward safe immediate pleasures.

A preschooler's ability to choose a larger, delayed reward over a smaller, immediate reward predicts development over the lifespan. ${ }^{1}$ Children who prefer delayed rewards develop more favorably in terms of cognitive and social competence, conduct and addictive behavior. ${ }^{1}$ Given such extensive correlates, the choice between options at different points in time-also termed intertemporal decision-making-has been widely investigated. The notion that a delay reduces a reward's subjective value (SV) has led to the concept of temporal or delay discounting (DD), ${ }^{2,3} \mathrm{a}$ measure of impulsive behavior. ${ }^{4}$ In other words, someone may find an immediate $10 €$ reward equally attractive as $20 €$ available in a month-both options have an equivalent SV for this person. Each individual discounts delayed rewards at a different rate, and based on a model assumed to underlie DD, this individual DD rate can be estimated from a set of choices between immediate and delayed rewards. A higher DD rate denotes steeper discounting and more impulsive choices. Individual differences in DD are stable, ${ }^{5}$ and DD rate is increased in adult addiction. ${ }^{6}$ Moreover, some findings indicate that DD is a predisposing factor for substance abuse, ${ }^{4,7,8}$ with at least one longitudinal analysis showing that DD predicts substance use. ${ }^{9}$
On the neural level, converging evidence points toward a single valuation system in the ventral striatum (VS)/nucleus accumbens (NAcc) and the ventromedial prefrontal cortex (vmPFC) representing the SV of delayed rewards in intertemporal choice paradigms. ${ }^{10-12}$ The neural representation of delayed reward SV has been intensely investigated. ${ }^{13-16}$ A recent study ${ }^{16}$ investigated differences in the SV representation during intertemporal choices in pathological gamblers and controls, replicating previous findings of increased DD rates in gamblers. Gambling severity was inversely correlated with delayed reward SV representations in the VS, vmPFC and midbrain. ${ }^{16} \mathrm{~A}$ corresponding pattern has been observed in smokers, who had lower striatal activation than non-smokers in anticipation of delayed rewards as compared with preference-matched immediate rewards. ${ }^{17}$

A crucial question concerns the mechanism by which individual differences in DD and the respective neural SV representation are attained. Although the degree of DD is partly heritable, ${ }^{18,19}$ little evidence exists to explain the remaining variability. However, some studies implicate that parenting practices might contribute to shaping DD. Reduced inhibitory control and steeper increases in alcohol and cigarette use are observed in the context of harsh or inconsistent parenting. ${ }^{20-22}$ Permissive parenting (that is, lacking guidance and consistency) has been related to impulsive choice in a delay of gratification paradigm. ${ }^{23}$ Particularly the samesex parent may have a role in impulsivity and substance use. ${ }^{24,25}$ More specifically, college students portraying their same-sex parent as permissive described themselves as more impulsive. ${ }^{25}$ Impulsivity in turn mediated the relationship between permissive same-sex parenting and increased past year alcohol use. ${ }^{25} \mathrm{~A}$

\footnotetext{
${ }^{1}$ Department of Systems Neuroscience, University Medical Center Hamburg-Eppendorf, Hamburg, Germany; ${ }^{2}$ Helen Wills Neuroscience Institute, University of California, Berkeley, CA, USA and ${ }^{3}$ Department of Psychology, Stanford University, Stanford, CA, USA. Correspondence: S Schneider, Department of Systems Neuroscience, University Medical Centre Hamburg-Eppendorf, Martinistrasse 52, Hamburg 20246, Germany.

E-mail: s.schneider@uke.uni-hamburg.de
}

Received 4 November 2013; revised 24 December 2013; accepted 23 February 2014 
recent study ${ }^{26}$ showed that after experiencing the experimenter as unreliable, children are less inclined to delay gratification. The notion that discounting is enhanced when the source of reward is perceived as unreliable has been supported in a different study. ${ }^{27}$ However, to our knowledge, no study has investigated how specifically parental delayed reward inconsistency relates to neural processing during intertemporal decision-making. Moreover, it is unclear whether experiences of unreliability generalize to discounting in a different, reliable context.

The present study investigated the link between delayed reward inconsistency and DD in healthy adolescents. An initial correlational analysis examined whether self-reported parental reward inconsistency was associated with DD and the concomitant neural SV representation, hypothesizing a particularly important role of the same-sex parent. ${ }^{25}$ As NAcc and vmPFC consistently encode $\mathrm{SV}^{28-30}$ analyses focused on these regions. Furthermore, in an experimental design, half of our sample experienced an 'unrelated' episode of repeatedly not receiving promised delayed rewards from a different investigator, resembling parental delayed reward inconsistency. The remaining participants consistently received delayed rewards, and we examined how this intervention modulated behavioral and neural indices of DD.

\section{MATERIALS AND METHODS}

\section{Participants}

The sample consisted of 48 healthy adolescents (13-15 years; Table 1). Participants were recruited in public schools with approval of the local school authority. Written informed consent was obtained from the parents and assent from the participants themselves. Procedures were approved by the local ethics committee. Exclusion criteria were neurological and serious physical conditions, claustrophobia, non-MR-compatible implants, indication of current suicidal thoughts, or positive screening for mental disorders. Two participants were excluded because of incidental findings. Even though complete data sets were acquired from 56 participants, 8 were excluded after the assessment. Three participants were excluded because requirements for post-intervention data reliability were not met (for example, incorrectly reported to have received all rewards). Participants were also excluded when they did not confirm to be able to provide reliable self-report data about both parents (two participants), if no discounting occurred (one participant), if screening for mental disorders (Supplementary Material) was positive (one participant), or if excessive current substance use was reported (that is, substance use on most of the days during the past 30 days; one participant).

\section{Questionnaires}

Using the well-validated Erziehungsstil-Inventar (parenting style inventory; $\mathrm{ESI}^{33}$ in both mother and father version, same-sex and opposite-sex reward inconsistency were assessed. More specifically, the sum score of three items (for example, 'It happens that my father promises me a reward and then forgets about it'; for the other items, please refer to the Supplementary Material) was calculated, with 0 indicating no inconsistency. Participants were requested to report alcohol, cigarette and cannabis use (Supplementary Table S1). As cigarette and cannabis use were rare, we focused on alcohol use to examine associations between substance use and impulsivity. Further information about questionnaires and neuropsychological testing are described in the Supplementary Material.

\section{Discounting}

The two-session fMRI discounting task conducted at both assessments $\left(T_{0}\right.$ and $\left.T_{1}\right)$, an adapted version of previous procedures, ${ }^{13,14}$ consisted of 84 trials per session (Figure 1). Both assessments included two discounting sessions representing different conditions (see Intervention section). Participants were instructed that all choices in the first session were hypothetical, while they would receive their choice of reward from a randomly selected second session trial. Further task details are described in the Supplementary Material.

\section{Intervention}

After $\mathrm{T}_{0} \mathrm{MRI}$ assessment (conducted by investigator $\mathrm{A}$ ), a new investigator (B) asked participants to participate in an unrelated study ('email study', the intervention; for an overview of the experimental design, see Supplementary Figure S1). Between $T_{0}$ and $T_{1}$, after participants had received the reward from $T_{0}$ discounting, they were required to complete a 2-week (experimentally irrelevant) 'homework' consisting of eight email reports about a rewarding experience of the day that made them proud. For these reports, they were promised gift certificates by investigator B. The control group (CON) received all certificates as indicated. However, the experimental group (EXP) was rewarded for the first, but not for further

Table 1. Characteristics of the total sample and the two groups.

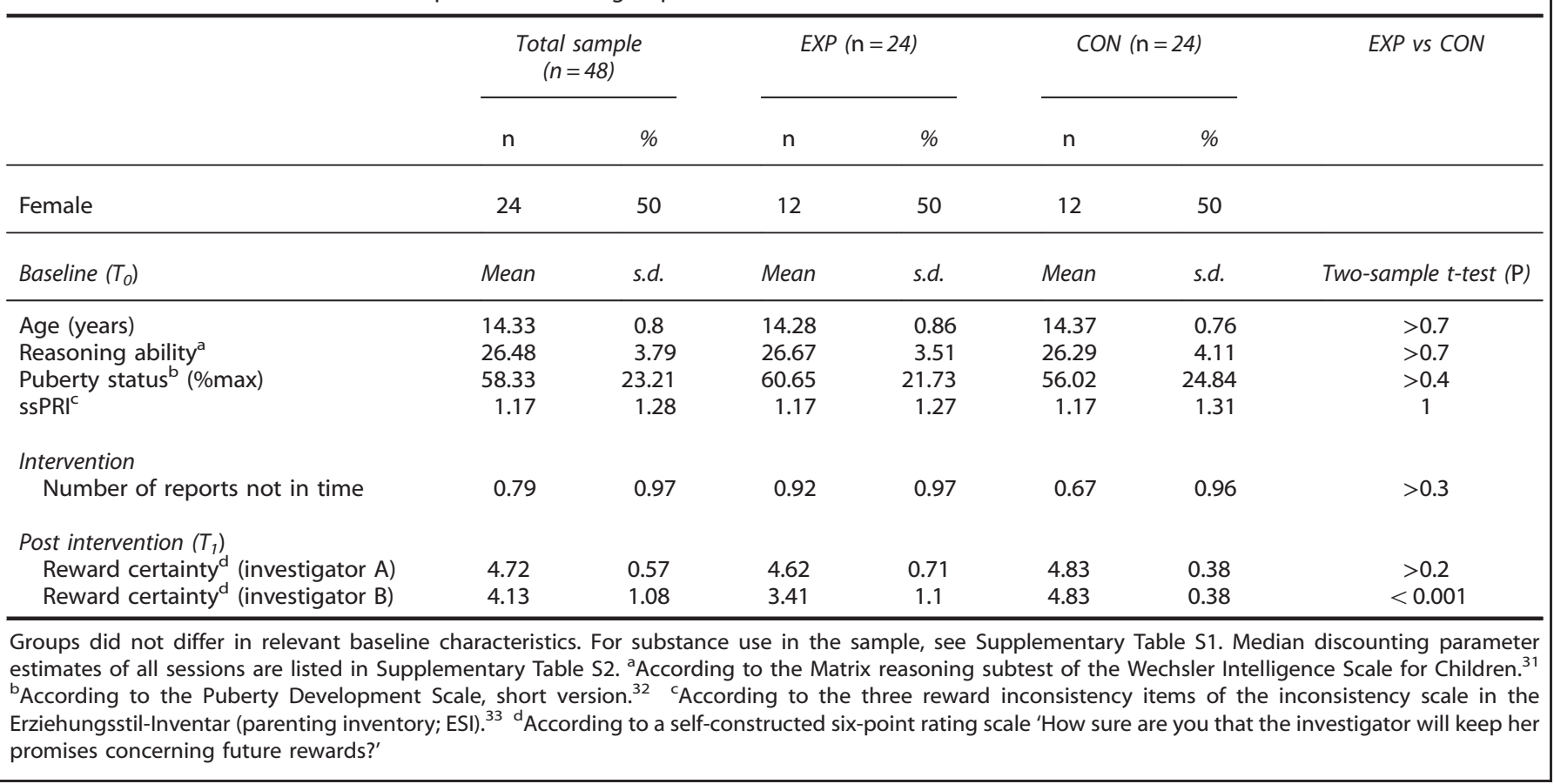




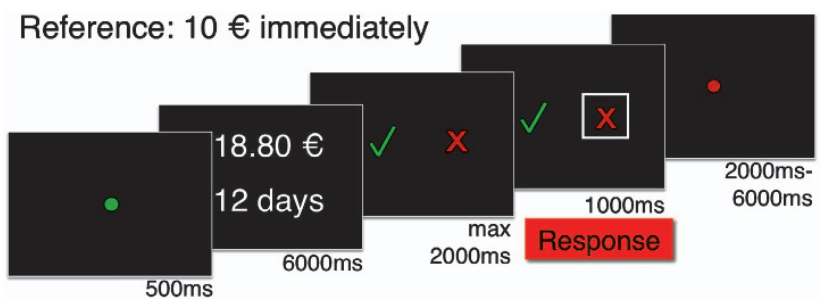

Figure 1. The time line of the discounting task adapted from Peters and Büchel. ${ }^{13,14}$ After a trial start cue, participants were required to decide whether they preferred the presented delayed reward over a fix $10 €$ immediate reward. Subsequently, they had to indicate their decision as quickly as possible (green checkmark: delayed, red cross: immediate). After decision feedback, a jitter interval preceded the next trial onset. For computational modeling of the participants' choices during the task, please refer to Supplementary Table S3 and Supplementary Figure S2.

reports. Groups did not differ in baseline characteristics (Table 1). While some participants had problems adhering to the instructed schedule, all participants eventually completed at least $85 \%$ of the reports. Critically, EXP participants were still missing certificates at $T_{1}$. The impression that the MRI study and the email study as well as respective investigators were completely unrelated was maintained throughout the experiment. However, it could still be argued that EXP participants would simply learn that rewards from all study investigators were uncertain, and that potential effects of the manipulation could be a mere consequence of this learning effect. For this reason, we included two conditions (hypothetical and real) in each DD assessment ( $T_{0}$ and $T_{1}$; see Discounting). If EXP participants would solely show expected effects because they considered rewards from investigator A equally uncertain as those from investigator $B$, this would only affect discounting for real rewards.

The $T_{1}$ assessment took place five weeks ( \pm 5 days) after $T_{0}$. After MRI discounting (conducted by Investigator A), participants rated their certainty to receive a promised delayed reward from each parent and each investigator $(A, B)$ on a one-item rating scale (see Supplementary Material). EXP participants then received withheld certificates and were completely debriefed. Further details are described in the Supplementary Text.

\section{Behavioral analyses}

Statistical analyses of the behavioral data were conducted using SPSS (SPSS, Chicago, IL, USA) and R (http://www.r-project.org/). A dualparameter model to estimate discounted value developed by Rachlin ${ }^{34}$ provided the best fit for the present data (for computational modeling, see Supplementary Material). In this model, an additional parameter $s$ is included into the standard hyperbolic discounting function accounting for differences in subjective time perception. ${ }^{35}$ As our hypotheses focused on discount rate, the additional parameter's contribution was removed from discount rate analyses by including it as a covariate to enhance sensitivity for individual differences. A square-root transformation was applied, ${ }^{36,37}$ accounting for skewed distributions of parameter estimates and their proximity to zero in some cases. For reasons of robustness, non-parametric correlations (Spearman's $\rho$ ) are reported.

\section{MRI and analyses}

MRI data were acquired using a 3-Tesla scanner (Siemens TRIO) located at the University Medical Centre Hamburg-Eppendorf, Hamburg, Germany, with a 32-channel head coil. Depending on reaction time, 420-454 volumes (mean: 430 volumes) were acquired in one session, taking $\sim 18 \mathrm{~min}$. Each volume comprised 41 slices aligned to the anterior-posterior commissure line $(2 \mathrm{~mm}$ thickness, $1 \mathrm{~mm}$ gap, TR $=2460 \mathrm{~ms}$, $\mathrm{TE}=25 \mathrm{~ms}, \mathrm{FOV}=216 \times 216 \mathrm{~mm}^{2}$, in-plane resolution $2 \times 2 \mathrm{~mm}^{2}$, GRAPPA factor 2). After functional imaging, high-resolution anatomical MR images were acquired (using a T1-weighted MPRAGE sequence).

All image analysis procedures were conducted using SPM8 (Wellcome Department of Cognitive Neurology, University College London). Functional images underwent slice-time correction before spatial realignment and unwarping. On the unsmoothed single-subject data, a first-level general linear model was constructed. The delayed option presentation and the response onset were included as event regressors (stick functions with the duration of 0 ). Regressors were convolved with the canonical hemodynamic response function.

SV of each delayed option ${ }^{34}$ was included as a parametric modulator of the delayed option presentation. This allowed us to investigate whether the BOLD signal associated with the onset of the delayed option co-varies with the SV of this option, that is, whether the presentation of a delayed option with a larger SV relates to a larger BOLD response. This parametric relationship between delayed reward SV and BOLD response is referred to as 'neural SV representation'.

Trials in which subjects responded too early/late were modeled separately. Contrast images for the SV representation across sessions were constructed for $T_{0}$ and $T_{1}$ as well as for the differential SV representation between sessions. The contrast ' $\left[S V\left(T_{0}\right.\right.$, session 1$)-S V\left(T_{0}\right.$, session 2)] - $\left[S V\left(T_{1} \text {, session } 1\right)-S V\left(T_{1} \text {, session2 }\right)\right]^{\prime}$ was constructed to investigate a potential time by group by session interaction.

$T_{0}$ and $T_{1}$ structural images were coregistered with the first functional scan and segmented with the voxel-based morphometry (VBM8) toolbox implemented in SPM8. From resulting gray and white matter segments, a custom template was created with DARTEL. Using individual flow-fields, contrast images were normalized to MNI space and smoothed with a 5$\mathrm{mm}$ Gaussian isotropic kernel. Normalizing and smoothing the contrast images instead of the entire time-series leads to a substantial saving of disk space and computation time, with virtually identical results on the second level. This is because the flow fields with the normalization parameters are estimated on the basis of the T1 structural images and only applied to functional images or contrast images, respectively. This procedure has been used regularly in our group. ${ }^{38-42}$

For correlational analyses, second-level one-sample $t$-tests were implemented. Same-sex/opposite-sex parent reward inconsistency (see Questionnaires) was included as respective covariate of interest. In order to exclude that results were confounded with socio-demographic variables, a separate control analysis was conducted, additionally including gender, age and reasoning ability (as an intelligence estimate) ${ }^{31}$ as nuisance covariates. For the experimental group analyses, second-level two-sample $t$-tests (EXP vs CON) were implemented.

Analyses were tested for two-tailed significance via F-contrasts, with one-tailed post hoc $t$-contrasts testing directionality, based on the same regions of interest as the initial two-tailed $F$-contrasts. Primary significance statements were based on the $F$-contrasts, and the $t$-contrasts were only to confirm the directionality of the effects. The threshold for significance was $P=0.05$, family-wise error corrected, using small-volume correction. Regions of interest were the bilateral NAcc (using masks from the Harvard-Oxford Cortical /Subcortical Structural Atlas (http://www.cma.mgh. harvard.edu/fsl_atlas.html), probability threshold $=0.5$ ) and vmPFC. Correction for the vmPFC was performed using a mask based on a recent metaanalysis, ${ }^{30}$ located at $x, y, z:(-15)-0,24-60,(-6)-(-21)$ in the left hemisphere and $0-9,26-45,(-3)-(-18)$ in the right hemisphere. Unless otherwise indicated, figures are displayed at a threshold of $P<0.005$, uncorrected, for visualization purposes, and projected onto the mean structural scan.

\section{RESULTS}

\section{Correlational analyses}

We first investigated whether discount rates differed between sessions (hypothetical, real). Discount rates computed on the basis of only hypothetical or real trials, respectively, revealed a high correlation (Spearman's $\rho=0.72, P<0.001$ ) and no differences (paired $t$-test, $P>0.4$ ). Correlational analyses therefore used discount rates derived from fitting the discounting model to data pooled across hypothetical and real trials.

Consistent with our hypothesis, same-sex parent reward inconsistency positively correlated with $\mathrm{T}_{0}$ discount rate $k$ $(\rho=0.34, P=0.020$; Figure 2a), in contrast to reward inconsistency by the opposite-sex parent $(P>0.4)$. In control analyses, we confirmed that including gender, age and reasoning ability ${ }^{31}$ as covariates did not affect the correlation, and that the correlation was specific to delayed reward inconsistency (see Supplementary Material). In addition, self-reported impulsivity ${ }^{43,44}$ also showed a trend-level positive association with reward inconsistency of the 

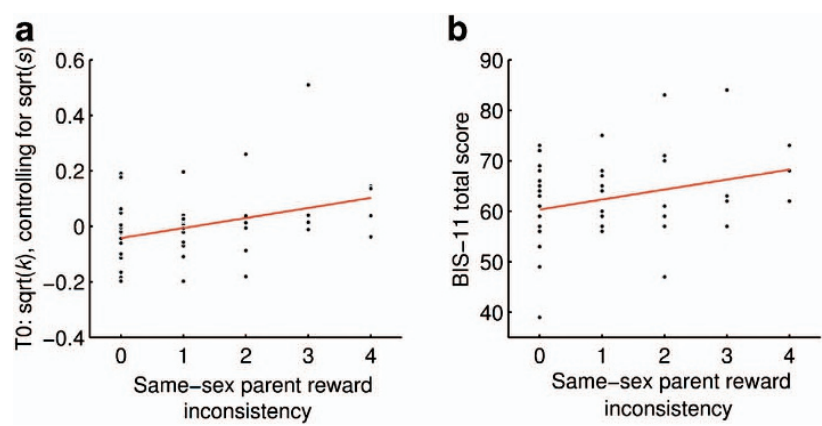

Figure 2. Correlational association between same-sex parent reward inconsistency (according to the Erziehungsstil-Inventar (parenting style inventory), ESI $)^{33}$ and two measures of impulsivity. (a) Association with the delay discount rate $k$, controlling for differences in subjective time perception represented by parameter $s$ (parameter estimates are square-root transformed; partial correlation between same-sex parent reward inconsistency and $k$, covarying for $s: P=0.020$ ). (b) Association with trait impulsivity as assessed by a German version of the Barratt Impulsiveness Scale-11 for adolescents (BIS- $11 ;, 43,44 P=0.078)$.

same-sex parent $(\rho=0.26, P=0.078$; Figure $2 b)$, but not with inconsistency of the opposite-sex parent $(P>0.7)$.

We next investigated indications of the well-established link between discount rate and alcohol use $\mathrm{e}^{45}$ in the present sample. While the correlation with past 30 days alcohol use did not reach significance $(P>0.1$, Bonferroni-corrected), the trend-level correlation with lifetime alcohol use $(\rho=0.33, P=0.075$, Bonferronicorrected) was explained when including gender, age and reasoning ability $(P>0.2$, Bonferroni-corrected). However, past
12 months alcohol use was positively associated with $k(\rho=0.47$, $P=0.002$, Bonferroni-corrected; Supplementary Figure S3), and this correlation was still significant when including gender, age and reasoning ability.

On the neural level, SV across $T_{0}$ sessions was robustly represented by the BOLD signal in bilateral NAcc $(x, y, z:-6,10$ -4 and $8,12,-4, F(1,47)=26.97$ and $F(1,47)=31.54$, both $P<0.001$, corrected; Figure 3a) and bilateral $\operatorname{vmPFC}(-6,26,-8$ and $4,44,-2$, $F(1,47)=24.72$ and $F(1,47)=27.73, P=0.003$ and $P=0.001$, corrected; Figure 3a). Post hoc t-contrasts confirmed that SV was positively associated with the BOLD signal $(t>4.97, P<0.002)$. As the NAcc and vmPFC SV representation did not differ between sessions ( $P>0.3$ and $P>0.2$, corrected), we continued to use the mean across sessions for the following analyses.

Consistent with our expectations, same-sex parent reward inconsistency was associated with the SV representations in the left NAcc $(-12,16-6, \quad F(1,46)=13.22, \quad P=0.014$, corrected; Figure $3 b)$ and left vmPFC $(-6,32,-18, F(1,46)=24, P=0.004$, corrected; Figure $3 \mathrm{~b}$ ). Including gender, age and reasoning ability as covariates of no interest in a control analysis did not explain these associations. A post hoc t-contrast confirmed that both associations were negative $(t(46)=3.64$ and $t(46)=4.9, P=0.007$ and $P=0.002$, corrected). Reward inconsistency by the oppositesex parent was not related to the SV representation in the NAcc $(P>0.2$, corrected), but there was a trend-level left vmPFC association $(-10,46,-18, F(1,46)=14.57, P=.069$, corrected). However, this association was explained when including gender, age and reasoning ability ( $P>0.1$, corrected).

\section{Experimental analyses}

We first examined whether the certainty to receive a delayed reward from the same-sex parent at $\mathrm{T}_{1}$ (as assessed by a one-item

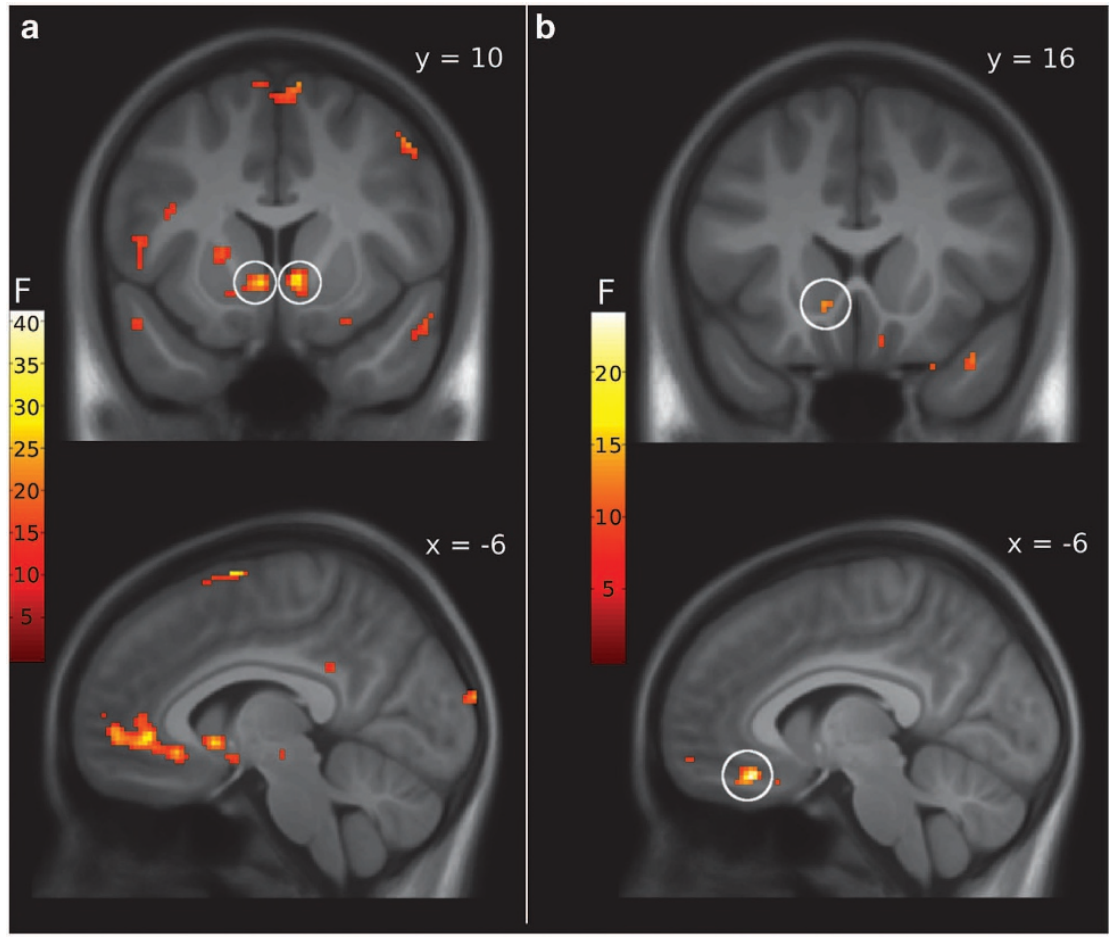

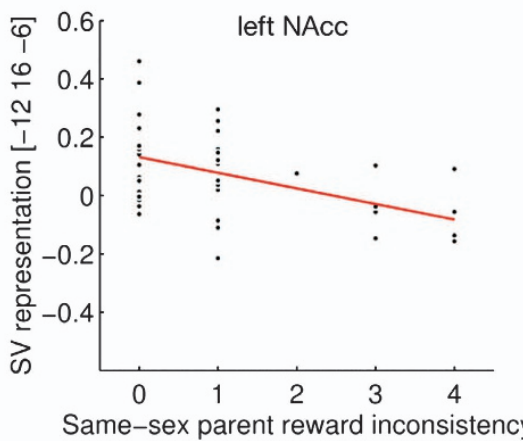

Same-sex parent reward inconsistency

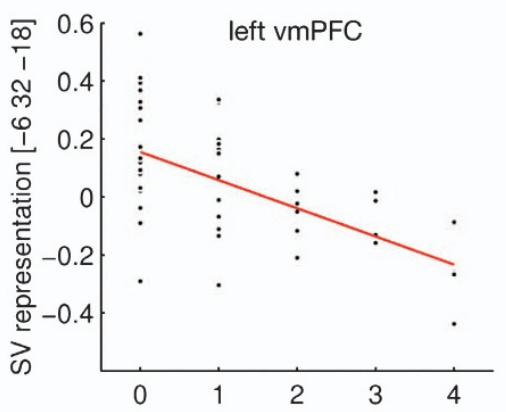

Same-sex parent reward inconsistency

Figure 3. (a) The neural representation of subjective delayed reward value at the baseline assessment $\left(\mathrm{T}_{0}\right)$. In the coronal view (upper panel), activation of the bilateral nucleus accumbens (NAcc; both $P<0.001$, corrected) is depicted, while the sagittal view (lower panel) shows the activation in the left ventromedial prefrontal cortex (vmPFC; $P=0.003$ and $P=0.001$, corrected). (b) Association between same-sex parent delayed reward inconsistency and the neural subjective value representation in the left NAcc (upper panel; $P=0.014$, corrected) as well as in the left vmPFC (lower panel; $P=0.004$, corrected). Scatter plots show individual parental inconsistency scores and estimates of the neural SV representation in the peak voxel (arbitrary units). The images in (a) are displayed with a threshold of $P<0.001$, uncorrected, and the images in (b) are displayed with a threshold of $P<0.005$, uncorrected, for visualization purposes. Color bars represent $F$ values. 


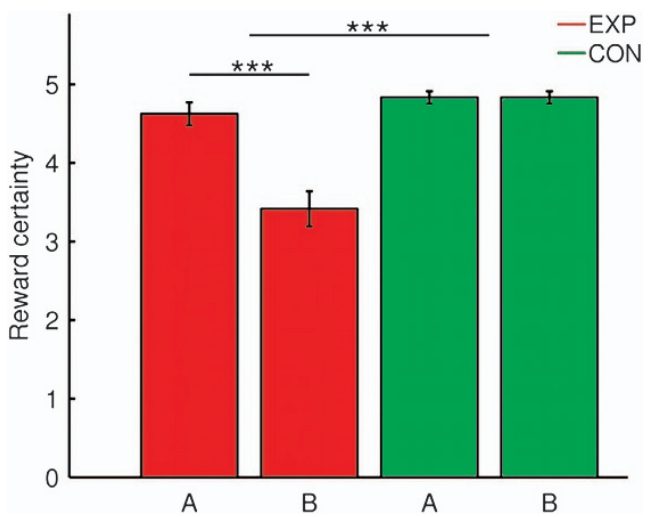

Figure 4. Ratings of subjective certainty to receive a promised delayed reward from investigator $A$ and investigator $B$ in the experimental group (EXP, $n=24)$ and the control group (CON, $n=24$; mean \pm s.e.m.). ${ }^{* * *} P<.001$.

rating scale, see Intervention section and Supplementary Material) was related to same-sex parent reward inconsistency at $T_{0}$ (as assessed by the ESI questionnaire). Consistent with these measures aiming at opposing constructs (reward inconsistency vs certainty to receive a delayed reward), they were strongly inversely correlated $(\rho=-0.49, P<0.001)$. Furthermore, $\mathrm{T}_{1}$ samesex parent reward certainty was negatively associated with $T_{0}$ discount rate $(\rho=-0.39, P=0.006)$. This finding replicates the link between same-sex parent delayed reward inconsistency and DD rate using a different measure than the ESI.

We then tested whether the intervention had successfully induced a between-investigator difference in reward certainty ratings in EXP (but not in CON) participants, which a significant interaction confirmed (2x2-factorial analysis of variance (ANOVA), $F(1,46)=33.64, P<0.001$; Figure 4$)$. Investigator $B$ was rated as less reliable than investigator A by EXP participants (paired $t$-test, $t$ $(23)=5.8, P<0.001$ ), but not by CON participants (paired $t$-test, $P=1)$. There was no group difference in reward certainty concerning investigator A (Table 1).

We next investigated a differential discount rate change between groups. Groups did not differ in baseline discount rate (two-sample $t$-test, $P>0.3$; for median parameter estimates, see Supplementary Table S2). In order to investigate the changes in discount rate in both groups, we conducted a group-by-time-bysession repeated measures ANOVA with $k$ as dependent variable. As the group-by-time-by-session interaction was not significant (ANOVA, $P>.1$ ), the group-by-time interaction was interpretable.
However, in contrast to our hypothesis, it was not significant (ANOVA, $P>0.2$ ).

On the neural level, we first investigated whether groups differed in the $T_{0} S V$ representation in the VmPFC and in the NAcc, which was not the case $(P>0.1)$. Also, session did not impact on the group-by-time interaction $(P>0.2)$. Thus, we examined the expected group-by-time interaction, with a significant result in the left NAcc $(-14,16,-8, F(1,46)=10.2, P=0.043$, corrected; Figure 5a and b), directly adjacent to the peak voxel showing the SV association with same-sex parent reward inconsistency at $T_{0}$ (Figure 5c). Post hoc $t$-contrasts showed an attenuated SV representation in EXP participants at $T_{1}(-14,16,-6, t(23)=3.1$, $P=0.039$; Supplementary Figure S4A and B) and no difference in CON participants $(P>.4)$. Control analyses showed that this result was not due to a non-existing $T_{0} S V$ representation in the CON group, as a significant NAcc SV representation was present in both groups at $\mathrm{T}_{0}$ (see Supplementary Material). No significant groupby-time interaction was observed in the vmPFC $(P>0.4)$.

Interestingly, despite being aware that in contrast to investigator A, investigator $B$ had withheld the largest part of their promised reward, some EXP participants $(n=6)$ did not differentiate between investigators in terms of reward certainty ratings. In attempt to explain these differences between EXP participants, we correlated non-adherence to schedule (the number of emails not sent in time) with intervention response (the normalized between-investigator differences in certainty ratings), yielding a significant negative correlation $(\rho=-0.56, \quad P=0.005$; Supplementary Figure S4C). Crucially, non-adherence was also inversely related to discount rate change (based on both hypothetical and real trials; $\rho=-0.56, P=0.006$; Supplementary Figure S4C). While the $T_{1}-T_{0}$ difference in the NAcc SV representation at the peak voxel was not associated with nonadherence $(P>0.1)$, there was a tentative negative association with intervention response $(\rho=-0.40, P=0.050$; Supplementary Figure S4D). We verified this result by additionally correlating intervention response with the mean difference of the voxels within the NAcc showing a significant attenuation from $T_{0}$ to $T_{1}$ (see Supplementary Material; $\rho=-0.42, P=0.039)$. These findings indicate that EXP participants who had more closely adhered to study requirements showed a stronger intervention response and an increase in discount rate. In turn, stronger intervention response related to a stronger attenuation in the NAcc SV representation.

\section{DISCUSSION}

Our combined correlational and experimental results indicate that inconsistent realization of delayed rewards attenuates the neural
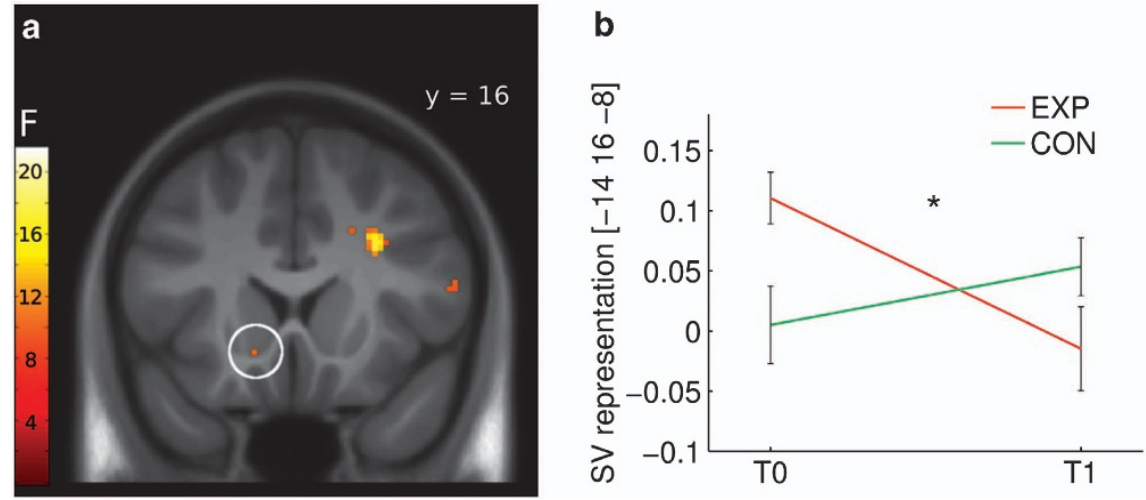

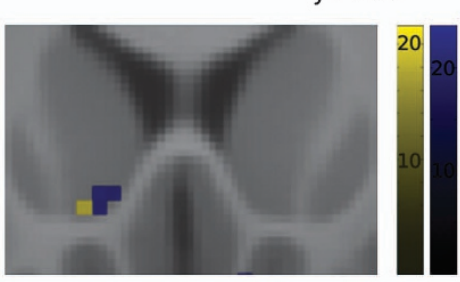

SV: group by time interaction

Correlation SV - same-sex parent reward inconsistency

Figure 5. (a) Group-by-time interaction of the left nucleus accumbens subjective value (SV) representation $(P=0.043$, corrected). (b) Estimates of the neural SV representation in the interaction peak voxel (arbitrary units; mean \pm s.e.m.). (c) The adjacent locations of the same-sex parent reward inconsistency association with the SV representation and the group-by-time interaction in the SV representation. Images are displayed with a threshold of $P<0.005$, uncorrected, for visualization purposes. Color bars represent $F$ values. 
SV representation in the NAcc and may contribute to impulsive choice. Specifically, self-reported delayed reward inconsistency by the same-sex parent was robustly associated with steeper DD as well as the neural SV representation in the NAcc and vmPFC. Experimentally induced reward inconsistency led to changes in the correlation between SV and the NAcc BOLD signal.

The correlational association between same-sex parent reward inconsistency and discount rate was robust, and it was further substantiated by the converging result obtained with the postintervention rating of parental reward certainty. Same-sex parent reward inconsistency was also trend-wise related to trait impulsivity. In line with previous findings, ${ }^{25}$ the reward inconsistency association with both facets of impulsivity (discount rate and trait impulsivity) was specific to the same-sex parent. This imbalance might be based on a preference for rewards from the same-sex parent in the context of emerging gender roles. Children preferentially imitate adult models of the same sex, ${ }^{46,47}$ and sex differences in toy choice have been documented as early as age one. ${ }^{48}$ The same-sex parent may therefore have a particularly important role in forming reward-related expectations and behavior. Notably, our findings do not indicate that the opposite-sex parent does not affect offspring impulsivity, but rather that we detected only a same-sex parent effect.

The correlation of same-sex parent reward inconsistency with discount rate was complemented by a negative association with the SV representation in the left NAcc and vmPFC. These neural effects do not simply mirror the behavioral findings. To elaborate, as SV is computed such that it predicts the choices, the neural activation correlated with SV-the neural SV representation-also relates to the choices. However, variations in the strength of the SV representation are independent of the choices. In other words, the discount rate reflects the tendency to choose immediate over delayed rewards, and the strength of the SV representation reflects the ability of neural activation to signal the value predicting these choices. Our findings indicate that in adolescents with more inconsistently rewarding same-sex parents, the left NAcc and VmPFC BOLD signal in response to the presentation of a delayed option covaries less with the SV of the delayed reward. In these adolescents, neural SV computations can explain less well whether a delayed option is accepted or rejected. Similar relationships have been observed between activation related to delayed rewards and smoking status ${ }^{17}$ or gambling severity. ${ }^{16}$ As an underlying mechanism, we suggest that breaking the contingency between expecting a delayed reward and actually receiving it disrupts the parametric relationship between the BOLD signal and choice behavior. Assuming that the BOLD signal in the NAcc and vmPFC is based on the expectancy of reward fulfillment, failure of reward fulfillment may blur the reward expectancy and thus the SV signal in the respective areas. While this mechanism is independent of the discount rate, it could-on the long run-facilitate impulsive choices. More simplified, if the reward system is less useful in signaling what to wait for, shortsighted aspects may gain more influence in the choice process.

Nonetheless, these associations on the behavioral and neural level do not provide causal evidence that experiencing reward inconsistency generates a preference for immediate rewards and attenuates the SV signal. Conversely, an experimental design allows addressing the issue of causality. In contrast to our hypotheses, the experimental results did not show a differential change in discount rate between EXP and CON. However, additional analyses showed that the more reliably EXP participants had adhered to study requirements, the stronger they responded to the intervention, and the more they showed the expected behavioral changes. Thus, inequity could be a crucial experience in the process of adjusting behavior as a consequence of not receiving anticipated rewards. In contrast to prior studies, ${ }^{26,27}$ we attempted to induce a general change in discount rate by an experience of unreliability and inequity (investigator B) that appeared unrelated to the discounting task (investigator A). While our intervention was not strong enough to induce this experience in all participants, a repeated disappointment caused by someone a child is closely identified with-for example, the same-sex parent-may produce such a general shift in behavior.

Whereas groups did not show a differential change in discount rate, their SV representation in the left NAcc changed differentially from $T_{0}$ to $T_{1}$, and this change was related to intervention response in the EXP group. Thus, consistent with our predictions, a reduction in certainty to receive a delayed reward may disturb delayed reward value processing. While in the correlational analyses, same-sex parent reward inconsistency was related to the SV representation in the NAcc and vmPFC, only the NAcc was affected by the intervention. Both regions have been widely implicated in the representation of SV. ${ }^{10,29,30,49}$ Nevertheless, some functional differences in the context of reward value have been proposed. Hare et $a .^{50}$ found that the medial orbitofrontal cortex coded the goal value of a stimulus (which denotes the abstract value of a stimulus independent of related costs, for example, a cue signaling a reward). In contrast, the NAcc signaled the prediction error, that is, was more active when the reward was higher than expected and less active when the reward was lower than expected. ${ }^{50}$ Only the NAcc was sensitive to effort cost in a paradigm using a fixed reward value. ${ }^{51}$ Considering these conceptualizations, the selective attenuation of the NAcc in the present study could be interpreted as the integrated cost of uncertainty, which is not necessarily reflected in choice behavior. This corresponds to the relation between NAcc SV representation change and reduced certainty to receive a reward from investigator $\mathrm{B}$.

It could be argued that any observed intervention effect depended on learning, such that EXP participants could have learned not to trust either investigator and therefore regarded rewards from the discounting task as uncertain. We consider this explanation unlikely. Only EXP participants distinguished between the consistent investigator $A$ and the inconsistent investigator $B$ in terms of reward certainty (Figure 4), and reward certainty concerning investigator A did not differ between groups. Moreover, if participants considered specifically rewards from the investigators uncertain, their processing of real rewards would change, but not their processing of hypothetical rewards. In the hypothetical session, participants were asked to indicate their general preference, and such a general preference should not depend on assumptions confined to rewards from the investigators. The lack of session differences indicates that in the EXP group, processing of delayed rewards had changed in a more general way. We thus exclude the possibility that the experimental effect is attributable to a perceived difference in the certainty to receive a reward from the $T_{1}$ discounting task.

In the light of impulsivity as a well-researched vulnerability factor for substance-related and addictive disorders, ${ }^{4}$ our findings may have essential implications for parenting as a target in addiction prevention. Even in the present sample, a correlation between impulsive behavior and alcohol use could be observed. Our results demonstrate that parents' repeated careless handling of reward promises may affect their children's tendency to make impulsive choices. Moreover, the experimentally induced change in the neural representation of delayed reward value indicates that the experience of delayed reward inconsistency might alter the contingency between the neural processing of delayed reward value and behavior.

Our study encompasses several limitations. First of all, as based on an adolescent sample, it is unclear how our findings relate to different age groups. While the correlational results appear robust, no general behavioral effect was induced by the intervention, and thus no causal link can be drawn between parental reward inconsistency and discount rate. However, in support of a partly 
causal relationship, prior findings show that the perception of unreliability undermines the will to wait for delayed rewards. ${ }^{26,27}$ Another issue is that we cannot exclude the possibility that the heritability of impulsivity has influenced the correlational results, as an impulsive parent might be prone to forgetting previously promised rewards. Nonetheless, our experimental results may speak against pure heritability because the neural changes induced by our intervention are in line with our hypotheses and the correlational results. Considering prior findings, ${ }^{26}$ it seems unlikely that repeatedly experiencing unreliability during childhood and adolescence will not have any effect on discount rate. Finally, although we replicated the link between discount rate and alcohol use in the present sample, a much larger sample would be needed to reliably examine relations between inconsistent parenting, impulsivity and substance use in depth.

In conclusion, our results give new insights into how parenting could shape individual differences in the neural representation of delayed reward SV and impulsivity. We propose that if children learn early that their investments do not pay off, they may develop a preference for immediately rewarding experiences. This could in turn predispose them to the later use of psychotropic substances. Future studies will have to extend these findings by delineating the mechanisms linking early-life reward experiences to neurocognitive markers of impulsivity.

\section{CONFLICT OF INTEREST}

The authors declare no conflict of interest.

\section{ACKNOWLEDGMENTS}

This work was supported by the German Research Foundation (DFG, SFB 936 and SFB TRR 58).

\section{REFERENCES}

1 Mischel W, Shoda Y, Rodriguez MI. Delay of gratification in children. Science 1989; 244: 933-938

2 Mazur J. An adjusting procedure for studying delayed reinforcement. Commons, M, Mazur, J, Navin, J. The Effect of Delay and Intervening Events on Reinforcement Value. Hillsdale, NJ, USA: Erlbaum, 1987, 55-73.

3 Samuelson PA. A note on measurement of utility. Rev Econ Stud 1937; 4: 155-161.

4 De Wit H. Impulsivity as a determinant and consequence of drug use: A review of underlying processes. Addict Biol 2009; 14: 22-31.

5 Kirby KN. One-year temporal stability of delay-discount rates. Psychon Bull Rev 2009; 16: 457-462.

6 Bickel WK, Miller ML, Yi R, Kowal BP, Lindquist DM, Pitcock JA. Behavioral and neuroeconomics of drug addiction: Competing neural systems and temporal discounting processes. Drug Alcohol Depend 2007; 90(Suppl 1): S85-S91.

7 Dalley JW, Everitt BJ, Robbins TW. Impulsivity, compulsivity, and top-down cognitive control. Neuron 2011; 69: 680-694.

8 Bickel WK, Koffarnus MN, Moody L, Wilson AG. The behavioral- and neuroeconomic process of temporal discounting: A candidate behavioral marker of addiction. Neuropharmacology 2014; 76: 518-527.

9 Audrain-McGovern J, Rodriguez D, Epstein LH, Cuevas J, Rodgers K, Wileyto EP. Does delay discounting play an etiological role in smoking or is it a consequence of smoking? Drug Alcohol Depend 2009; 103: 99-106.

10 Kable JW, Glimcher PW. The neural correlates of subjective value during intertemporal choice. Nat Neurosci 2007; 10: 1625-1633.

11 Kable JW, Glimcher PW. An 'as soon as possible' effect in human intertemporal decision making: Behavioral evidence and neural mechanisms. I Neurophysiol 2010; 103: 2513-2531.

12 Monterosso JR, Luo S. An argument against dual valuation system competition: Cognitive capacities supporting future orientation mediate rather than compete with visceral motivations. J Neurosci Psychol Econ 2010; 3: 1-14.

13 Peters J, Büchel C. Overlapping and distinct neural systems code for subjective value during intertemporal and risky decision making. J Neurosci 2009; 29: 15727-15734.

14 Peters J, Büchel C. Episodic future thinking reduces reward delay discounting through an enhancement of prefrontal-mediotemporal interactions. Neuron 2010; 66: $138-148$.
15 Prévost C, Pessiglione M, Météreau E, Cléry-Melin M-L, Dreher J-C. Separate valuation subsystems for delay and effort decision costs. J Neurosci 2010; 30: 14080-14090.

16 Miedl SF, Peters J, Büchel C. Altered neural reward representations in pathological gamblers revealed by delay and probability discounting. Arch Gen Psychiatry 2012; 69: 177-186.

17 Luo S, Ainslie G, Giragosian L, Monterosso JR. Striatal hyposensitivity to delayed rewards among cigarette smokers. Drug Alcohol Depend 2011; 116: 18-23.

18 Anokhin AP, Golosheykin S, Grant JD, Heath AC. Heritability of delay discounting in adolescence: A longitudinal twin study. Behav Genet 2011; 41: 175-183.

19 MacKillop J. Integrating behavioral economics and behavioral genetics: Delayed reward discounting as an endophenotype for addictive disorders. J Exp Anal Behav 2013; 99: 14-31.

20 Luyckx K, Tildesley EA, Soenens B, Andrews JA, Hampson SE, Peterson M et al. Parenting and trajectories of children's maladaptive behaviors: A 12-year prospective community study. J Clin Child Adolesc Psychol 2011; 40: 468-478.

21 Moilanen KL, Shaw DS, Dishion TJ, Gardner F, Wilson M. Predictors of longitudinal growth in inhibitory control in early childhood. Soc Dev 2009; 19: 326-347.

22 Pears K, Capaldi DM, Owen LD. Substance use risk across three generations: The roles of parent discipline practices and inhibitory control. Psychol Addict Behav 2007; 21: 373-386.

23 Mauro CF, Harris YR. The influence of maternal child-rearing attitudes and teaching behaviors on preschoolers' delay of gratification. J Genet Psychol 2000; 161: 292-306.

24 Patock-Peckham JA, Cheong J, Balhorn ME, Nagoshi CT. A social learning perspective: A model of parenting styles, self-regulation, perceived drinking control, and alcohol use and problems. Alcohol Clin Exp Res 2001; 25: 1284-1292.

25 Patock-Peckham JA, Morgan-Lopez AA. College drinking behaviors: Mediational links between parenting styles, impulse control, and alcohol-related outcomes. Psychol Addict Behav 2006; 20: 117-125.

26 Kidd C, Palmeri H, Aslin RN. Rational snacking: Young children's decision-making on the marshmallow task is moderated by beliefs about environmental reliability. Cognition 2013; 126: 109-114.

27 Michaelson L, de la Vega A, Chatham CH, Munakata Y. Delaying gratification depends on social trust. Front Psychol 2013; 4: 355.

28 Knutson B, Taylor J, Kaufman M, Peterson R, Glover G. Distributed neural representation of expected value. J Neurosci 2005; 25: 4806-4812.

29 Bartra O, McGuire JT, Kable JW. The valuation system: A coordinate-based metaanalysis of BOLD fMRI experiments examining neural correlates of subjective value. Neurolmage 2013; 76: 412-427.

30 Levy DJ, Glimcher PW. The root of all value: A neural common currency for choice. Curr Opin Neurobiol 2012; 22: 1027-1038.

31 Wechsler D. Wechsler Intelligence Scale for Children (WISC-IV). 4th edn. London, UK: Pearson Assessment, 2004

32 Petersen AC. Adolescent development. Annu Rev Psychol 1988; 39: 583-607.

33 Krohne HW Pulsack A. Das Erziehungsstil-Inventar (ESI): Manual. 2nd edn. Weinheim, Germany: Beltz Test, 1995.

34 Rachlin H. Notes on discounting. J Exp Anal Behav 2006; 85: 425-435.

35 Zauberman G, Kim BK, Malkoc SA, Bettman JR. Discounting time and time discounting: Subjective time perception and intertemporal preferences. J Marketing Res 2009; 46: 543-556.

36 Peters J, Miedl SF, Büchel C. Formal comparison of dual-parameter temporal discounting models in controls and pathological gamblers. PLoS One 2012; 7: e47225.

37 Ballard K, Knutson B. Dissociable neural representations of future reward magnitude and delay during temporal discounting. Neuroimage 2009; 45: 143-150.

38 Schneider S, Peters J, Bromberg U, Brassen S, Miedl SF, Banaschewski T et al. Risk taking and the adolescent reward system: A potential common link to substance abuse. Am J Psychiatry 2012; 169: 39-46.

39 Peters J, Bromberg U, Schneider S, Brassen S, Menz M, Banaschewski T et al. Lower ventral striatal activation during reward anticipation in adolescent smokers. Am J Psychiatry 2011; 168: 540-549.

40 Schneider S, Brassen S, Bromberg U, Banaschewski T, Conrod P, Flor $\mathrm{H}$ et al. Maternal interpersonal affiliation is associated with adolescents' brain structure and reward processing. Transl Psychiatry 2012; 2: e182.

41 Menz MM, Rihm JS, Salari N, Born J, Kalisch R, Pape HC et al. The role of sleep and sleep deprivation in consolidating fear memories. Neurolmage 2013; 75: 87-96.

42 Büchel C, Brassen S, Yacubian J, Kalisch R, Sommer T. Ventral striatal signal changes represent missed opportunities and predict future choice. Neuroimage 2011; 57: 1124-1130.

43 Patton JH, Stanford MS, Barratt ES. Factor structure of the Barratt impulsiveness scale. J Clin Psychol 1995; 51: 768-774.

44 Hartmann AS, Rief W, Hilbert A. Psychometric properties of the German version of the Barratt Impulsiveness Scale, Version 11 (BIS-11) for adolescents. Percept Mot Skills 2011; 112: 353-368. 
45 Lejuez CW, Magidson JF, Mitchell SH, Sinha R, Stevens MC, de Wit H. Behavioral and biological indicators of impulsivity in the development of alcohol use, problems, and disorders. Alcohol Clin Exp Res 2010; 34: 1334-1345.

46 Bussey K, Bandura A. Influence of gender constancy and social power on sexlinked modeling. J Pers Soc Psychol 1984; 47: 1292-1302.

47 Grace DM, David BJ, Ryan MK. Investigating preschoolers' categorical thinking about gender through imitation, attention, and the use of self-categories. Child Dev 2008; 79: 1928-1941.

48 Servin A, Bohlin G, Berlin L. Sex differences in 1-, 3-, and 5-year-olds' toy-choice in a structured play-session. Scand J Psychol 1999; 40: 43-48.

49 Peters J, Büchel C. Neural representations of subjective reward value. Behav Brain Res 2010; 213: 135-141.

Supplementary Information accompanies the paper on the Translational Psychiatry website (http://www.nature.com/tp)
50 Hare TA, O'Doherty J, Camerer CF, Schultz W, Rangel A. Dissociating the role of the orbitofrontal cortex and the striatum in the computation of goal values and prediction errors. J Neurosci 2008; 28: 5623-5630.

51 Botvinick MM, Huffstetler S, McGuire JT. Effort discounting in human nucleus accumbens. Cogn Affect Behav Neurosci 2009; 9: 16-27.

(1) $\Theta$ This work is licensed under a Creative Commons AttributionNonCommercial-NoDerivs 3.0 Unported License. To view a copy of this license, visit http://creativecommons.org/licenses/by-nc-nd/3.0/ 